\title{
Quaderni
}

QUADERNI Communication, technologies, pouvoir

73 | Automne 2010

La métropole parisienne entre récits, paroles et échanges

\section{Avant-propos : pour une approche communicationnelle de la métropole parisienne}

\section{Dominique Pagès}

\section{(2) OpenEdition}

Journals

Édition électronique

URL : http://journals.openedition.org/quaderni/439

DOI : 10.4000/quaderni.439

ISSN : 2105-2956

Éditeur

Les éditions de la Maison des sciences de l'Homme

Édition imprimée

Date de publication : 5 octobre 2010

Pagination : 5-8

Référence électronique

Dominique Pagès, « Avant-propos : pour une approche communicationnelle de la métropole parisienne », Quaderni [En ligne], 73 | Automne 2010, mis en ligne le 05 octobre 2012, consulté le 30 avril 2019. URL : http://journals.openedition.org/quaderni/439; DOI : 10.4000/quaderni.439 


\section{$D$ ossier}

\section{avant-propos :}

pour une

approche

communica-

tionnelle de

la métropole

parisienne

Dominique
Pagès

Maître de Conférences

CELSA, GRIPIC

Université de Paris Sorbonne
La fabrique symbolique des métropoles au fil de leur mise en récits, des échanges qui la constituent et des prises de parole de leurs habitants (pour en constater les métamorphoses et pouvoir se les approprier au quotidien, pour en commenter les leurres et les opacités) constitue le sujet de ce dossier qui privilégie ainsi une approche interdisciplinaire.

L'affirmation de la métropole parisienne passe par sa capacité à s'écrire et à se dire, à s'imaginer et à se partager. Un déploiement d'images et de textes plus ou moins inédits et contradictoires participe du devenir métropole qui s'impose à l'agglomération parisienne et appelle des interprétations actualisées tant la métropolisation est un processus complexe, à la fois global et localisé, à la fois politique et ordinaire. Mettant à l'épreuve le mythe de Paris mais aussi la forme, l'organisation et les modalités de vie de la Capitale, la métropolisation demande un renouvellement des analyses urbaines, des lectures symboliques et donc un dialogue entre des approches contrastées.

Au-delà de leur diversité thématique et disciplinaire, les articles de ce numéro affirment la possible place des individus, de leur parole, de leurs échanges, de leur capacité à agir dans la fabrique métropolitaine. Partant du constat que l'individu métropolitain est ou plutôt sera de moins en moins un habitant passif, soumis à une autorité claire, stable, lisible et unique, chaque auteur aborde à partir de son angle de vue la possibilité de ce dire et de cet agir, de ce possible investissement des espaces et des temps de la métropole parisienne, mais aussi de toute métropole à vivre et à rendre plus vivable encore. Ce dossier part d'un choix volontairement restrictif pour aborder l'immense 
chantier de la métropolisation de Paris : envisageant la réforme du Grand Paris par le biais des récits, des paroles et des échanges, il cherche à actualiser le regard porté de manière parfois hégémonique par les urbanistes, les architectes et les spécialistes de la gouvernance en abordant la métropolisation par le biais de ses enjeux communicationnels. La métropole parisienne cherche sa propre définition, sa forme et sa singularité ; cette entreprise doit se situer par rapport aux approches d'autres villes, des grandes métropoles du monde mais sans s'y identifier. De là, l'importance de la construction plurielle, évolutive, contrastée du récit qui la conduit et des débats qui la dialoguent.

Mon article qui ouvre le numéro pose l'hypothèse d'une nécessaire mise en tension des récits métropolitains en présence pour que la dynamique métropolitaine se déploie : j'envisage ainsi successivement le patient récit mais quelque peu perturbateur de Paris Métropole, celui, épique, du Grand Paris et enfin celui, épars et contradictoire, mais se densifiant, de « la Métropole des individus » dans une perspective dialectique plutôt qu'oppositionnelle. Leurs formes et leurs logiques, leurs modes d'apparition et de diffusion différent mais les envisager ensemble apparaît indispensable pour prendre en compte la complexité métropolitaine et éviter toute velléité d'unification et de mise en ordre symbolique de celle-ci.

L'article de Cynthia Ghorra-Gobin met en perspective les principaux éléments du discours sur le processus de métropolisation en s'appuyant aussi bien sur les corpus français qu'anglo-américains. La métropolisation se présente comme un concept, voire même un «nouveau » paradigme toutes les fois qu'il est question de ville, de développement urbain et d'aménagement du territoire. Ce concept est principalement utilisé par les géographes et les économistes qui s'interrogent aussi bien sur les dynamiques spatiales à l'origine d'une extension de la ville sans aucune notion de limite que sur les ressorts de cette dynamique liée à la globalisation de l'économie. Suite à l'impulsion par la nouvelle phase du capitalisme, indissociable des usages et diffusions des technologies de communication et d'information, les sciences sociales parlent moins de ville que de « territoire métropolitain » ou de « ville-région ». L'auteur souligne par ailleurs les 'nouveaux' coûts sociaux et écologiques engendrés par ce processus.

Frédéric Gilli reprend les grandes étapes du récit qui ont jalonné la construction des termes d'une controverse sur le devenir métropolitain de Paris. Il pose l'hypothèse que le projet Paris Métropole serait avant tout un objet transitionnel qui aura permis de fixer les attentions et les discours sur "la nécessité d'un changement que personne n'osait nommer ». Il lui semble que, derrière la querelle sémantique, l'énoncé même de la notion de métropole est une condition pour avancer et transformer les débats en actualisant l'ancienne problématique du Grand Paris. En cela, la démarche Paris Métropole serait une approche de rupture, tant dans la méthode, la philosophie que dans les moyens. La notion et le projet « Paris Métropole » sont ainsi étudiés à partir de leur vertu transformatrice.

Gilles Rayssac questionne les possibles conditions de l'émergence d'un citoyen métropolitain, «le grand absent du projet Grand Paris ». La construction de la métropole ne peut selon lui 
faire l'impasse de la prise en compte du citoyen, de l'habitant, de l'usager et du passant. La métropolisation serait ainsi le creuset d'une nouvelle forme d'exercice de la citoyenneté, pouvant modifier la relation traditionnelle qui s'est établie entre les élus, les « techno-experts » et le citoyen. Dans quelle mesure les citoyens peuventils définir leur cadre de vie ? Comment passer d'une logique d'administration des territoires et d'exploitation de leurs ressources à une logique de ménagement des parties prenantes ? Cet article pose concrètement la question de la gouvernance métropolitaine et de ses dispositifs.

Gustavo Gomez-Mejia poursuit par un état des lieux actualisé des formes dominantes de représentations de la métropole parisienne sur Internet. À partir d'un corpus de sites web, il interroge "les réalités discursives que coproduisent les dispositifs et les acteurs dans leur rapport aux différents marqueurs symboliques qui font métropole ». Déployant ainsi une lecture critique du projet métropolitain au prisme de Google, il en questionne les formulations qui « déterminent la circulation et la lisibilité ». Faisant le constat d'une prépondérance des discursivités prétendant à une expertise métropolitaine, il se tourne vers la place de la parole des habitants : celle que lui octroient les acteurs publics, institutionnels et experts ; celle effective des habitants, au fil des forums et des réseaux sociaux. $\mathrm{Si}$, de manière générale, les écarts entre le web institutionnel et le web vernaculaire tendent à se réduire, il ne semble pas qu'il en soit ainsi pour la question métropolitaine.

Emmanuelle Lallement interroge le rôle levier que l'échange marchand, ses situations, ses lieux et temps jouent ou pourraient jouer dans la métropolisation. Elle questionne la tension actuelle entre les discours dénonçant enseignes internationales et centres commerciaux de périphérie comme autant de facteurs d'uniformisation et les discours qui encensent les espaces marchands comme «l'âme de la ville », Paris étant liée plus que toute autre Capitale à son identité marchande. Après avoir esquissé une cartographie de la diversité des espaces marchands de la capitale, de leur statut, de leur localisation et temporalités, elle étudie le rôle structurant des commerces tant dans le renouvellement urbain que dans l'inscription de Paris dans un réseau de grandes métropoles marchandes. Selon elle, une certaine métropolisation est bien à l'œuvre dans l'organisation des marchés, celle-ci semblant dépasser le conflit entre uniformisation marchande et singularité locale.

Dans une perspective plus ethnographique, Sophie Corbillé s'intéresse au vécu métropolitain, à cette forme de vie urbaine complexe dans laquelle on trouve à la fois : "l'expression d'un mode de vie », "l'instauration d'un ordre social urbain » et l'attachement fort au «local» mais aussi le fait de « vivre une expérience ». Partant d'une enquête menée dans les quartiers gentrifiés du Nord-Est de Paris, elle se propose de montrer combien l'expérience métropolitaine s'appuie sur des lieux « branchés » et sur de nouvelles centralités fonctionnant sur une dynamique compétitive de la concurrence et de l'attractivité. Mais son article cherche surtout à mettre en évidence la persistance actuelle de la thématique de la frontière dans les paroles recueillies et, de là, la difficile émergence d'une appartenance commune entre les différents territoires de la métropole et donc d'une identité métropolitaine véritablement citadine. 
Enfin, l'entretien mené avec l'adjoint au Maire de Paris, chargé de Paris Métropole, Pierre Mansat précise l'analyse des évolutions du projet. Après avoir rappelé l'inscription progressive de Paris Métropole dans l'agenda politique, réaffirmé ses valeurs puis pointé les éléments de méthodes retenues pour faire naître « une conscience métropolitaine » et pour permettre l'émergence d'un lieu d'élaboration collective de politiques, Pierre Mansat précise son rôle d'impulsion et sa manière de faire de la politique dans le domaine de l'urbain mais aussi du culturel et de l'économique. Constatant l'absence de représentation médiatique claire et enracinée du territoire métropolitain, il rappelle la politique de communication mise en œuvre avec sa délégation et revient sur le rôle de son blog, véritable «plateforme de la construction métropolitaine ». Enfin, il pointe sa responsabilité politique et celle du maire de Paris à aider les habitants à s'impliquer dans une citoyenneté future.

Ce numéro, s'il ouvre ainsi un champ de recherches pour les sciences de l'information et de la communication, esquisse aussi quelques pistes pour les sciences politiques : la nécessité d'aborder la question de la gouvernance mais en se centrant plus finement sur le processus de décision en œuvre (dans la filiation des travaux de Lucien Sfez); le besoin d'études plus poussées sur la « difficile concordance des temps entre les logiques et les temporalités en pratique dans l'administration, celles en usage dans la politique, celles enfin propres aux acteurs économiques $»^{1}$. Ainsi, la métropolisation «dans tous ses états » semble augurer des travaux interdisciplinaires de longue haleine.

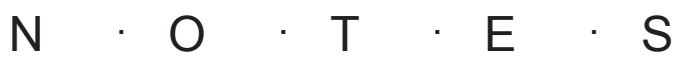

1. Pistes explicitées grâce à l'attentive lecture de Serge Graziani qui, avec Emmanuel Taiëb, fut le premier lecteur de ce dossier. Je les remercie tous deux. Merci aussi aux deux patients traducteurs de la plupart des résumés, Kyle Schneider et Philip Schneider. 